\title{
Extraskeletal myxoid chondrosarcoma in the lung: asymptomatic lung mass with severe anemia
}

\author{
Qianjun Zhou ${ }^{1,5^{*}}$, Guangzhong Lu², Aiqun $\mathrm{Liu}^{3}$ and Tadasu Kohno ${ }^{4}$
}

\begin{abstract}
Extraskeletal myxoid chondrosarcoma (EMC) is a rare soft-tissue sarcoma, which primarily occurs deep in the extremities, especially in skeletal muscle, or tendon. EMC of the pleura has been described, however, no case of primary EMC arising from lung has been previously reported. We describe herein, a 51-year-old Asian female initially manifested with signs of severe anemia who presented with a lung mass unrelated to pleura that was morphologically typical EMC, with strong immunoreactivity for vimentin and NSE. Two weeks after resection, the anemia was cured. The patient continued with follow-up, without sign of abnormality 32 months after operation. Virtual slides: The virtual slides for this article can be found here: http://www.diagnosticpathology.diagnomx.eu/vs/ 2882199847396682
\end{abstract}

Keywords: Extraskeletal myxoid chondrosarcoma, Lung, Immunohistochemistry, Anemia

\section{Background}

Extraskeletal myxoid chondrosarcoma (EMC) was first reported by Enzinger and Shiraki in 1972 as a rare soft-tissue sarcoma, which primarily occurs deep in the extremities, especially in skeletal muscle, or tendon [1]. Unusual locations include tongue, retroperitoneum, spine, intracranium, testis, inguinal region, synovium, mammary gland, and labium.

We report the clinicopathological features of a female patient with EMC of the lung initially manifested with asymptomatic lung mass with severe anemia determined by the evidence of histological and immunohistochemical examination.

\section{Case presentation}

A 51-year-old Asian woman, who was a social worker, nonsmoker, was admitted to the Department of Hematology in August 2009 because of severe anemia and emaciation without cough, sputum, and hemoptysis. Physical examination revealed marked sign of anemia

\footnotetext{
* Correspondence: qianjunz@buffalo.edu

'Department of General thoracic surgery, Shanghai First People's Hospital, Shanghai Jiao Tong University School of Medicine, Shanghai 200080, P. R. China

${ }^{5}$ Department of Microbiology and Immunology, State university of New York at Buffalo, Buffalo, NY 14214, USA

Full list of author information is available at the end of the article
}

but failed to find any masses in the soft tissue of extremities or pelvis through palpation. A computed tomography $(\mathrm{CT})$ scan disclosed a $41 \times 33 \mathrm{~mm}$ parenchymal mass on apico-posterior segment of left upper lobe, CT value was 26 Hounsfield unit (HU). The patient had a 10 -year history of left lung nodule by chest $\mathrm{x}$-ray, however, no films were available currently. Whole blood cell count was characterized as microcytic hypochromic anemia, with RBC $2.67 \times 10^{12} / \mathrm{L}, \mathrm{Hb} 54 \mathrm{~g} / \mathrm{L}$. Serum iron was $2.2 \mu \mathrm{mol} / \mathrm{L}$, total iron binding capacity $74 \mu \mathrm{mol} / \mathrm{L}$. Serum IgG $18.3 \mathrm{~g} / \mathrm{L}$, and a serum protein electrophoresis examination found no monoclonal protein. Tumor markers such as carcinoembryonic antigen (CEA) and neuron specific enolase (NSE) were all negative. Bone marrow smear and biopsy showed normocellularity with lower intracellular iron. Bronchoscopic examination with biopsy, brushing and washing found no abnormality. A bone scan was negative for other lesions. There were no metastatic signs in brain and abdomen by CT scan. After the therapy of iron supplement and red blood cell transfusion, $\mathrm{Hb}$ was slightly elevated. The patient was transferred for surgery on October, and thoracic CT scan showed the tumor was $55 \times 48 \mathrm{~mm}$, with CT value 34 $\mathrm{HU}, 54 \mathrm{HU}$ in contrast enhancement scanning (Figure 1). After anemia had been cured by transfusion, left exploratory thoracotomy was planned. Operation revealed 


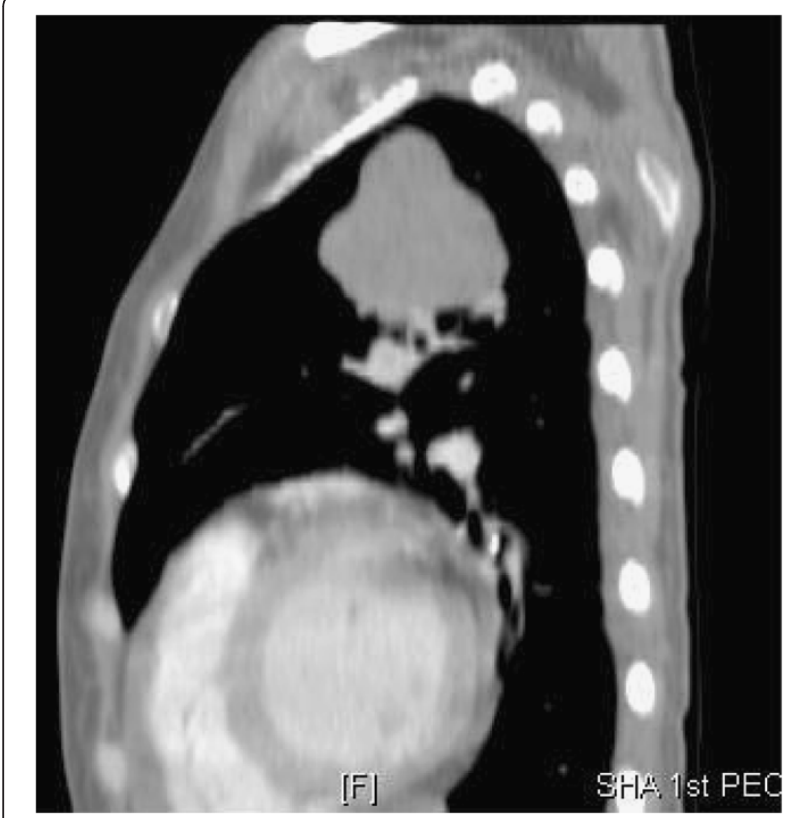

Figure 1 Preoperative radiological features: large mass involving the left upper lobe.

a peripheral pulmonary mass on left upper lobe without pleural indentation or swollen lymph node in hilum and mediastinum, and the patient underwent left upper lobectomy with systemic lymph node dissection. The patient recovered smoothly and was discharged in a good condition without anemia after two weeks. Follow-up continued every three month after operation by chest $\mathrm{CT}$ scan and $\mathrm{CBC}$. There was no sign of abnormality 32 months after operation.

Grossly, the left upper lobe measured $20 \mathrm{~cm} \times 10.5 \mathrm{~cm} \times$ $4 \mathrm{~cm}$, and the lesion was located in lung parenchyma 2 $\mathrm{cm}$ away from the resection margin of left upper bronchus. On cut surface, the tumor measured $5 \mathrm{~cm} \times 5 \mathrm{~cm} \times$ $3.2 \mathrm{~cm}$ in size, pale gray, soft, well demarcated, lobulated with a gelatinous texture.

Microscopic examination showed the characteristic features of EMC, including a well-circumscribed, multilobulated architecture separated by incomplete fibrous septae, relatively uniform oval to short spindle shaped cells arranged in short anastomosing strands and cords embedded in abundant myxoid matrix (Figure 2). The cells were characterized by hyperchromatic nucleus and tiny nucleolus, some of which showed grooves (Figure 3 ). There were a large number of plasma cells and a few lymphocytes in fibrous septae to form the compact zone (Figure 4). No hyaline cartilage was seen. Mitotic figures were $1 / 50 \mathrm{HPF}$. No infiltration into adjacent lung tissue was seen.

Special stains revealed glycogen in the cytoplasm of the tumor cells, while the surrounding extracellular

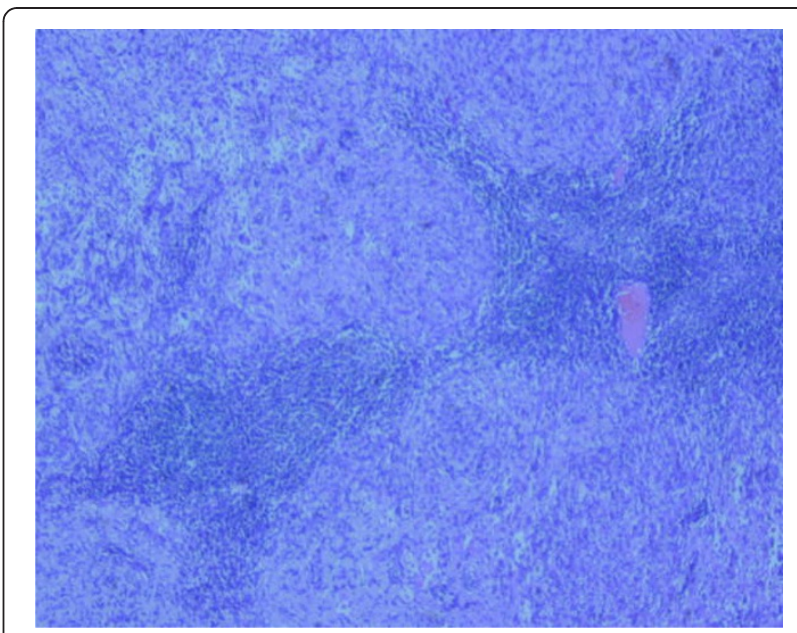

Figure 2 Low magnification shows the well-circumscribed tumor with multinodular architecture. $\times 40$.

matrix was positive with Alcian blue stain. By immunohistochemical study, vimentin and NSE were strongly expressed in the tumor cells (Figure 5, 6), while S-100 protein was weak and focal (Figure 7). The other markers including cytokeratin, epithelial membrane antigen (EMA), P63, smooth muscle actin (SMA), muscle specific actin (MSA), desmin, KP1, calretinin were all negative. IFN- $\beta$ was immunopositive in the tumor cells.

\section{Discussion}

EMC has mainly been reported in the areas of orthopedics, neurosurgery, and otolaryngology [1]. As with our case, the characteristic features of well-circumscribed, multilobulated configuration with incomplete fibrous septae, oval to spindle shaped cells arranged in short

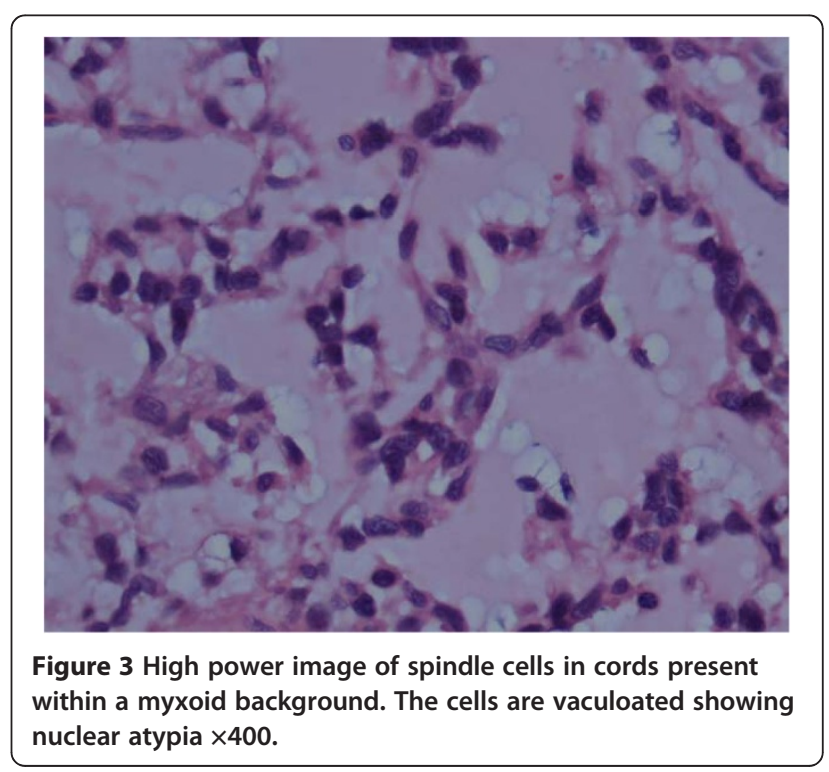




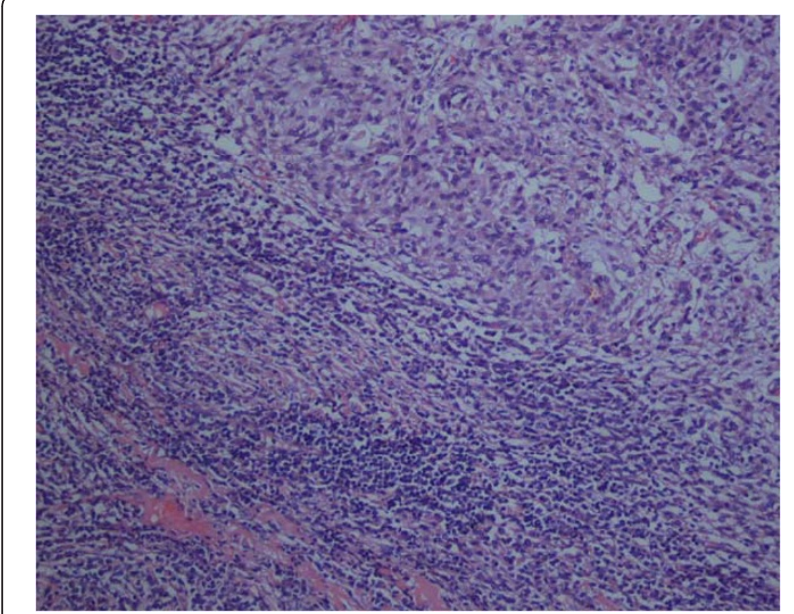

Figure 4 Medium power image illustrates the plasma cells and lymphocytes surrounding the tumor.

anastomosing strands embedded in abundant myxoid matrix and typical histochemical findings favored the diagnosis of EMC [2]. Since the lesion located at the periphery of the lung, fine needle aspiration biopsy (FNAB) should be initially done to help diagnosis. Although cytologic characteristics are not decisive for diagnosis, the presence of intranuclear cytoplasmic inclusions, grooves and cytoplasmic vacuolization could be suggestive to EMC diagnosis [3]. S-100 protein was initially found in most EMC, but recent studies proved that less than $20 \%$ of EMC are S-100 positive [4]. In some cases of EMC, there is neuroendocrine differentiation [5]. A specific chromosomal translocation $\mathrm{t}(9 ; 22)(\mathrm{q} 22 ; \mathrm{q} 12)$ leading to a fusion gene, EWS-CHN has been found in $75 \%$ EMC cases [6], and another fusion gene TAF2NCHN caused by $\mathrm{t}(9 ; 17)(\mathrm{q} 22 ; \mathrm{q} 11.2)$ translocation has

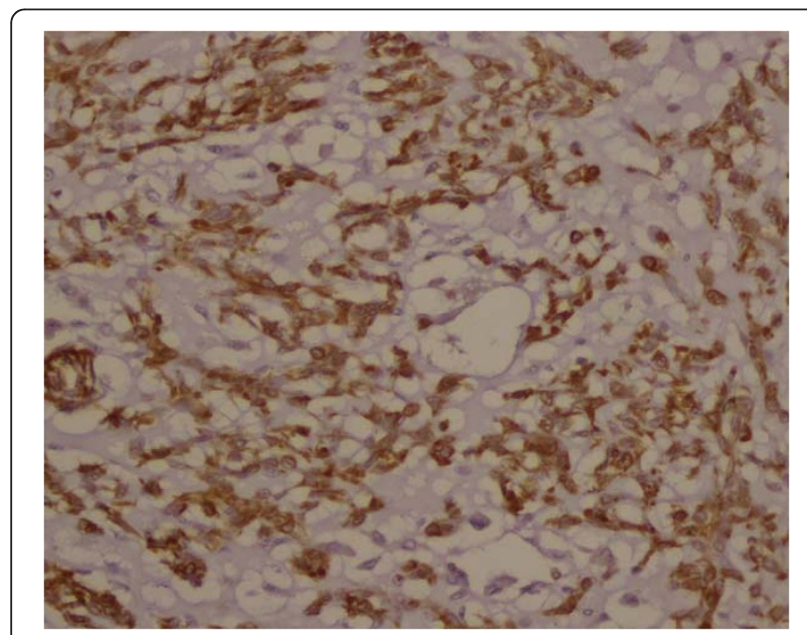

Figure 5 Photomicrographs shows the strong expression of Vimentin in tumor cells.

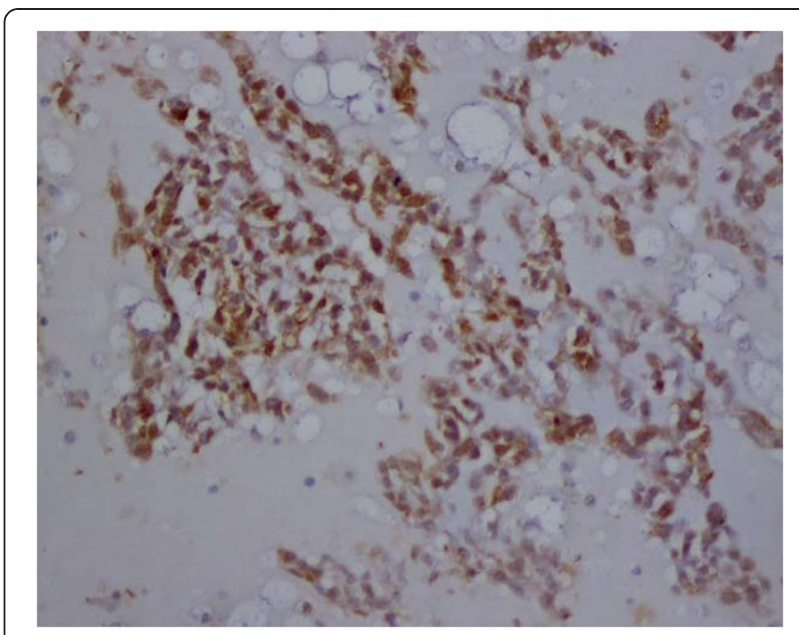

Figure 6 The tumors are strongly immunopositve to neuronspecific enolase.

recently been identified [5]. Although primaries should not have difficulty in diagnosis for experienced pathologists, the morphologic differential diagnosis in our case are parachordoma, chordoma, soft tissue chondroma, extraskeletal mesenchymal chondrosarcoma,as well as pulmonary metastases of EMC. Parachordoma is an indolent soft tissue tumor predominantly in the extremities and trunk, occasionally in the chest wall. The diagnosis is excluded because there were no typical histopathologic and immunohistochemical features of parachordoma such as plasma cell-like appearance of the tumor cells with chondrometaplasia myoepithelium, and expression of cytokeratin, and EMA. Chordoma appears as lobulated tumors, with cord pattern tumor cells scattering within abundant myxoid stroma. The diagnosis is ruled out by a lack of physaliferous cells, and no

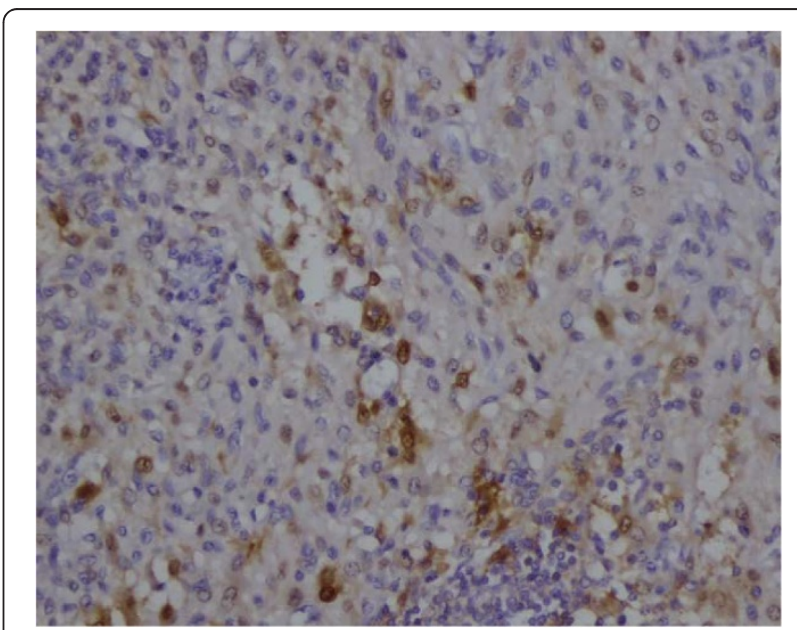

Figure 7 S-100 protein expression was weak and focal in the tumor. 
expression of cytokeratin. Soft tissue chondroma composed of small cells with hyaline cartilage lobule which lies near the joints of extremities, and S-100 is uniquely positive. Extraskeletal mesenchymal chondrosarcoma arising in lung [7], chest wall, pleural and mediastinum has been reported. The lack of small undifferentiated cells surrounding narrow vascular spaces in a haemangiopericytoid (haemangiopericytoma-like) pattern with mature cartilage island would exclude extraskeletal mesenchymal chondrosarcoma. As lung is the most frequent site of metastases in EMC, it is necessary to differetiate primary EMC in lung from lung metastases of EMC. However, it was not difficult to exclude lung metastases of EMC originated in the other parts of the body after comprehensive clinical evaluation and CT scan, with particular attention to the distal parts of the extremities. There was not any evidence of tumor in the body other than in the lung of the patient. Extraosseous (extramedullary) plasmacytoma (EMP) is defined as localized plasma cell neoplasms arising in tissues other than bone, and EMP in lung has been reported [8]. In our case, there are abundant plasma cells and aggregated lymphocytes in tumor fibrous septae and adjacent lung tissue which might be the anti-tumor reaction of immune system. As to EMP, the neoplastic cells are similar to normal plasma cells with very few lymphocytes. Last, our case should also be differentiated from primary pulmonary sarcomas. Pulmonary myxoid liposarcoma is multinodular-architecture in myxoid stroma with Vimentin and S-100 positive which is similar to EMC, however, lack of lipoblasts and plexiform capillary network exclude the diagnosis [9]. Immunostains can be helpful to rule out rhabdomyosarcoma, leiomyosarcoma. Primary and metastatic chondrosarcoma of lung have been documented, and the multilobular neoplasm with chondroid and myxoid matrix which is positive to Vimentin and S-100, negative for epithelial markers might cause the confusion with EMC [10]. In this case, absence of predominant chondromatous lesion helps to exclude chondrosarcoma. Pulmonary carcinosarcoma is ruled out for lack of carcinomatous component [11].

Although EMC is most common in the soft tissues of the extremities, in fact, the primary site can be anywhere in the body [1]. To our knowledge, no case of EMC arising in lung has been reported to date. Primary EMC of the pleura has been described by Goetz et al. [12]. From the CT scan, a parenchymal mass of left upper lobe can be found with distinct margin from the intact visceral pleura, which were also proved by microscopic examination on cut section of tumor. We suggest that the origin might be primitive mesenchymal cell, or associated with the tracheobronchial cartilage $[13,14]$.

Anemia is one of the characteristics in this patient which has not been reported previously in EMCs.
Patients afflicted with malignancy often develop anemia, and some cytokines, particularly TNF- $\alpha$, TGF- $\beta$,IFN- $\beta$, IL-1,are found increasing in many malignant diseases $[15,16]$. The function of these cytokines is related to retention of iron in the reticuloendothelial system, gastrointestinal tract and liver and exert inhibitory effects on erythroid precursors [17]. As in our case, expression of IFN- $\beta$ in tumor cells, significant in excessive type IFN signaling, inhibits erythropoiesis through decreased $\mathrm{Bcl}-$ XL expression level and enhanced apoptosis of erythroblasts [18]. Also, negative bone marrow cytology rules out occurrence of primary hematological malignancies, such as multiple myeloma. It is intriguing that the anemia was corrected just after the removal of the tumor, suggesting that the cause of anemia relies on the tumor itself.

EMC shows a tendency to affect patients beyond their fifth decade [19]. EMC was initially viewed as a lowgrade sarcoma with prolonged and indolent clinical course, but recent studies found its unfavorable prognosis with high rate of recurrence and metastases [20]. Significant in the past history was that our 51-year-old patient had a left lung nodule examined by routine chest $\mathrm{x}$-ray check for around 10 years, which was in accordance with those previous reports. Since EMC has a high rate of recurrence and metastases, and the death caused by tumor often occurs in a long postoperative period, sometimes more than 10 years after diagnosis, so we suggest that frequent and long-term follow up is needed for our patient.

\section{Conclusion}

Our case is the first case of EMC located in the lung. It highlights the possibility and difficulty of diagnosis of this rare tumor in the lung, especially in the patients with history of long-term and slowly-growing lung nodule. Characteristic histological features supported by immunohistochemical reactivities, help to diagnose EMC of the lung, although it is exceedingly rare in this location.

\section{Consent}

Written informed consent was obtained from the patient's relatives for publication of this case report and any accompanying images.

\section{Competing interests}

The authors declare that they have no competing interests.

\section{Authors' contributions}

QZ and GL equally contributed to this article. QZ, GL and KT conceived and designed the study, QZ and GL wrote the manuscript. QZ and AL participated in patient's management. GL made the pathological explorations. All authors read and approved the final manuscript.

\section{Author details}

'Department of General thoracic surgery, Shanghai First People's Hospital, Shanghai Jiao Tong University School of Medicine, Shanghai 200080, P. R. 
China. ${ }^{2}$ Department of Pathology, Shanghai First People's Hospital, Shanghai Jiao Tong University School of Medicine, Shanghai 200080, P. R. China. ${ }^{3}$ Department of Radiology, Shanghai First People's Hospital, Shanghai Jiao Tong University School of Medicine, Shanghai 200080, P. R. China. ${ }^{4}$ Department of General thoracic surgery, Toranomon Hospital, Tokyo 1058470, Japan. ${ }^{5}$ Department of Microbiology and Immunology, State university of New York at Buffalo, Buffalo, NY 14214, USA.

Received: 13 June 2012 Accepted: 13 August 2012

Published: 28 August 2012

\section{References}

1. Enzinger FM, Shiraki M: Extraskeletal myxoid chondrosarcoma. An analysis of 34 cases. Hum Pathol 1972, 3(3):421-435.

2. Goh YW, Spagnolo DV, Platten M, Caterina P, Fisher C, Oliveira AM Nascimento AG: Extraskeletal myxoid chondrosarcoma: a light microscopic, immunohistochemical, ultrastructural and immunoultrastructural study indicating neuroendocrine differentiation. Histopathology 2001, 39(5):514-524.

3. Kumar R, Rekhi B, Shirazi N, Pais A, Amare P, Gawde D, Jambhekar N: Spectrum of cytomorphological features, including literature review, of an extraskeletal myxoid chondrosarcoma with $\mathrm{t}(9 ; 22)(\mathrm{q} 22 ; \mathrm{q} 12)$ (TEC/ EWS) results in one case. Diagn Cytopathol 2008, 36(12):868-875.

4. Lucas DR, Fletcher CD, Adsay NV, Zalupski MM: High-grade extraskeletal myxoid chondrosarcoma: a high-grade epithelioid malignancy. Histopathology 1999, 35(3):201-208.

5. Harris M, Coyne J, Tariq M, Eyden BP, Atkinson M, Freemont AJ, Varley J, Attwooll C, Telford N: Extraskeletal myxoid chondrosarcoma with neuroendocrine differentiation: a pathologic, cytogenetic, and molecular study of a case with a novel translocation $\mathrm{t}(9 ; 17)(\mathrm{q} 22 ; \mathrm{q} 11.2)$. Am J Surg Pathol 2000, 24(7):1020-1026.

6. Hirabayashi $Y$, Ishida $T$, Yoshida MA, Kojima T, Ebihara $Y$, Machinami $R$, Ikeuchi T: Translocation (9;22)(q22;q12). A recurrent chromosome abnormality in extraskeletal myxoid chondrosarcoma. Cancer Genet Cytogenet 1995, 81(1):33-37.

7. Rocca M, Vanel D, Couanet D, Caillaud JM, Brugiere L: [Pulmonary mesenchymatous chondrosarcoma in children. Report of 2 cases and review of the literature]. J Radiol 1988, 69(5):329-332.

8. Zuo Z, Tang Y, Bi CF, Zhang WY, Zhao S, Wang XQ, Yang QP, Zou LQ, Liu WP: Extraosseous (extramedullary) plasmacytomas: a clinicopathologic and immunophenotypic study of 32 Chinese cases. Diagnostic pathology 2011, 6:123.

9. Uchikov A, Poriazova E, Zaprianov Z, Markova D: Low-grade pulmonary myxoid liposarcoma. Interactive cardiovascular and thoracic surgery 2005, 4(5):402-403.

10. Panelos J, Voulgaris S, Michos E, Doukas M, Charalabopoulos K, Batistatou A: Chondrosarcoma of the spine: a rare case with unusual presentation. Diagnostic pathology 2006, 1:39.

11. Olobatoke AO, David D, Hafeez W, Van T, Saleh HA: Pulmonary carcinosarcoma initially presenting as invasive aspergillosis: a case report of previously unreported combination. Diagnostic pathology 2010, 5:11.

12. Goetz SP, Robinson RA, Landas SK: Extraskeletal myxoid chondrosarcoma of the pleura. Report of a case clinically simulating mesothelioma. Am J Clin Pathol 1992, 97(4):498-502.

13. Daniels AC, Conner GH, Straus FH: Primary chondrosarcoma of the tracheobronchial tree. Report of a unique case and brief review. Arch Pathol 1967, 84(6):615-624.

14. Pescarmona E, Rendina EA, Venuta F, Pisacane A, Baroni CD: Myxoid chondrosarcoma of the mediastinum. Appl Pathol 1989, 7(5):318-321.

15. Moliterno AR, Spivak JL: Anemia of cancer. Hematol Oncol Clin North Am 1996, 10(2):345-363.

16. Bokemeyer C, Oechsle K, Hartmann JT: Anaemia in cancer patients: pathophysiology, incidence and treatment. Eur J Clin Invest 2005, 35(Suppl 3):26-31.

17. Jongen-Lavrencic M, Peeters HR, Vreugdenhil G, Swaak AJ: Interaction of inflammatory cytokines and erythropoeitin in iron metabolism and erythropoiesis in anaemia of chronic disease. Clin Rheumatol 1995, 14(5):519-525.

18. Mizutani T, Tsuji K, Ebihara Y, Taki S, Ohba Y, Taniguchi T, Honda K: Homeostatic erythropoiesis by the transcription factor IRF2 through attenuation of type I interferon signaling. Exp Hematol 2008, 36(3):255-264.

19. Kawaguchi S, Wada T, Nagoya S, Ikeda T, Isu K, Yamashiro K, Kawai A, Ishii T, Araki N, Myoui A, et al: Extraskeletal myxoid chondrosarcoma: a MultiInstitutional Study of 42 Cases in Japan. Cancer 2003, 97(5):1285-1292.

20. Antonescu CR, Argani P, Erlandson RA, Healey JH, Ladanyi M, Huvos AG: Skeletal and extraskeletal myxoid chondrosarcoma: a comparative clinicopathologic, ultrastructural, and molecular study. Cancer 1998, 83(8):1504-1521.

\section{doi:10.1186/1746-1596-7-112}

Cite this article as: Zhou et al:: Extraskeletal myxoid chondrosarcoma in the lung: asymptomatic lung mass with severe anemia. Diagnostic Pathology 2012 7:112.

\section{Submit your next manuscript to BioMed Central and take full advantage of:}

- Convenient online submission

- Thorough peer review

- No space constraints or color figure charges

- Immediate publication on acceptance

- Inclusion in PubMed, CAS, Scopus and Google Scholar

- Research which is freely available for redistribution

Submit your manuscript at www.biomedcentral.com/submit
C Biomed Central 